\title{
CONVENIENCE OF RUNNING-GEAR HEALTH MONITORING SYSTEMS TO REDUCE UNAVAILABILITY IN METROPOLITAN RAILWAYS
}

\author{
RICCARDO LICCIARDELLO ${ }^{1}$, ALESSANDRO SAPIENZA ${ }^{1}$, JUAN MORENO GARCÍA-LOYGORRI ${ }^{2}$, \\ JULIÁN MARTIIN JARILLO ${ }^{2}$ \& JOSÉ ANTONIO CHOVER ÁLVAREZ-MONTESERIIN ${ }^{2}$ \\ ${ }^{1}$ SAPIENZA University of Rome DICEA, Italy \\ ${ }^{2}$ Metro de Madrid, Spain
}

\begin{abstract}
Condition monitoring of the running gear of metro trainsets has been receiving increasing interest in recent years due to its potential for cost reduction and safety assurance. In fact, condition-monitoring systems (CMS) may be used (a) for the early identification of component faults (health monitoring), and (b) for in-service load monitoring. This paper describes the part of the research work performed in the SHIFT2RAIL-funded RUN2Rail project addressed to use (a). The focus is on the exploitation of the CMS to reduce the unavailability of trainsets and/or of service disruptions. This fits into the current general trend for railways towards condition-based maintenance (CBM) of assets, which has become a sector-wide need. Various technologies are available or under development for this purpose. It is assumed here that monitoring of health indicators is performed on a number of wheelsets, gearboxes and suspension elements of a case study trainset type of Metro de Madrid's fleet. The convenience of installing, or not, the systems is assessed based on the observed component failure rates, as input to a bespoke fault-tree analysis (FTA) approach. The probability of occurrence of events that may lead to trainset or service unavailability is taken as top event of the fault tree; it is calculated with different assumptions regarding the probability of detection (POD) of the monitoring systems and the probability that a given component fault will generate unavailability. The corresponding variation of unavailability costs (with/without CMS) is quantified through appropriate penalty costs. The main results are the methodology per se, with indications of which indicators should be monitored as an input to this type of convenience assessment. The method and the results are valuable for metropolitan railway operators in assessing the convenience of adopting condition monitoring systems in their new or existing rolling stock.
\end{abstract}

Keywords: condition monitoring system (CMS), health monitoring system (HMS), metro, railway, operating costs, unavailability.

\section{INTRODUCTION}

As for most complex technological systems, the running gear of metro trainsets undergoes complex maintenance. Moreover, running gear - composed of several items such as wheelsets, axle-boxes, primary and secondary suspension elements, bogie frame - is also safety-critical. Even a minor failure in an item, and its consequent fault state, can cause unavailability of the trainset for passenger service and withdrawal from service at a terminal station, or even worse at an intermediate station. The unavailability, and the hazards, may be particularly severe depending on the nature of the failure (e.g. a wheel flat versus a broken wheel). Corrective maintenance, i.e. "carried out after fault recognition and intended to restore an item into a state in which it can perform a required function" EN 13306 [1], does not generally prevent service unavailability events, which are added to the inevitable technical unavailability of the trainset or a part of it while the item is being restored e.g. in the workshop. Preventive maintenance, "intended to assess and/or to mitigate degradation and reduce the probability of failure of an item", is thus a necessary part of the overall maintenance plan. In the past, such maintenance was most often predetermined maintenance, "carried out in accordance with established intervals of time or 
number of units of use but without previous condition investigation". Inevitably, "healthy" items are replaced with this approach, leading to inefficiency which is nowadays being reduced with condition-based maintenance $(\mathrm{CBM})$, that is "preventive maintenance which includes assessment of physical conditions, analysis and the possible ensuing maintenance actions". A particularly attractive form of CBM from in this sense is predictive maintenance, "carried out following a forecast derived from repeated analysis or known characteristics and evaluation of the significant parameters of the degradation of the item". From the authors' perspective, railways are still "over-maintaining" their rolling stock and the transition to CBM is not an option but a mandatory action.

The condition assessment for CBM "may be by operator observation, and/or inspection, and/or testing, and/or condition monitoring of system parameters...". Thus, condition monitoring of the running gear of metro trainsets has been receiving increasing interest in recent years. Condition monitoring systems (CMS) on the trainset are designed to monitor key system parameters by means of a sensor network and measurement-chain conditioning devices (e.g. power, signal acquisition and processing, communications within the trainset and with outside systems, data recording etc., see Jarillo et al. [2]). Monitored system parameters are for example accelerations, strain/stress, displacements, pressures, angular velocities, temperatures ( $\mathrm{Li}$ et al. [3]). Such parameters may be correlated with the so-called health state the component/item, in which case they may be used as health indicators $(\mathrm{Xu}$ and $\mathrm{Xu}$ [4]). Monitored health indicators may be used as a basis for CBM, hence the use of the alternative expression health monitoring systems (HMS) for such applications. In addition to the use identified above, CMS (not necessarily HMS) also serve other purposes such as safety assurance, or in-service load monitoring for various purposes (Velletrani et al. [5]).

In a fault state, the item has lost partially or entirely its ability to perform a required function due to a failure (EN 13306 [1]). It is either in an "up state", or "available" (able to perform, including the "degraded state", with reduced ability to perform but with acceptable reduced performance), or in a "down state", or "unavailable", i.e. unable to perform. The down state relates to the "unavailability" of the item. An example of indicator to quantify unavailability $Q$ is the ratio of the down time $(D T)$ to the total time of a given period (that is the sum of the so-called up time and the down time): $Q=D T /(U T+D T)$. The $D T$ includes the time needed for preventive maintenance and the time in service in the down state. Availability depends on the combined aspects of reliability (how often does a failure occur?) and maintainability (how long does it take to restore?).

Unavailability of trainset items often generates unavailability of the transport service. These are the cases of interest in this research. At the moment, there are no universally accepted definitions and categorisation for this type of unavailability. In particular, it is interesting to note that for a transport service a degraded state (e.g. delays due to a trainset failure) would generally be understood as unavailability to some degree, differently from the unavailability of an item.

The idea behind the research work described here is that the use of a HMS for CBM should imply an early identification, or even a prediction, of item faults, in order to reduce the overall duration of service unavailability over a given reference period. The possibility for the HMS to do so depends on how early faults are identified: (1) before they create a safety hazard (but perhaps too late to avoid service unavailability); (2) before they create a service disruption (but perhaps too late to avoid delays); or (3) early enough to plan corrective maintenance in a way as to minimise the trainset's downtime (and avoiding entirely any consequence on service). This is related to a "probability of detection (POD)" of the fault by the HMS. 
Installing an HMS should be worthwhile if its detection capabilities are satisfactory, and if the entity of service unavailability in the current situation, dependent most of all on the observed item failure rates, is relevant enough.

Since in the RUN2Rail project in which this work was funded, technology concepts were proposed for various HMS/CMS, the objective was to present a methodology to assess the convenience of the RUN2Rail systems for applications such as that of Metro Madrid. The case study presented is not meant to be exhaustive in the cost calculation details but intends to provide a methodology and some early results to go on with the discussion on condition monitoring.

A first step to reach the objective was to explore existing methods for convenience assessment (Section 2). The case study described in Section 3 was approached with a novel methodology based on fault tree analysis (FTA) and described in Section 4. Under the assumptions described in Section 5 numerical results were obtained (Section 6) were obtained which led to the conclusions and recommendations of Section 7.

\section{STATE-OF-THE-ART}

The literature now abounds in descriptions of techniques for CMS and HMS. An excellent description of techniques is given in Li et al. [3]. The two main stages of on-board HMS are classified as data acquisition and data analysis. For data acquisition, inertial sensors (e.g. accelerometers) are widely used because of small size, low cost, low power consumption and robustness. Displacement sensors, noise sensors and heat sensors are also used. The signals are conditioned by suitable devices. Data analysis is performed through two main signal processing approaches: model-based and signal based. The former use mathematical methods whose running-dynamics-related outputs are compared with real-time measured outputs to identify faults. The latter extract fault-related characteristics from the measured signals in various ways, including time-domain, frequency-domain, time-frequency approaches and correlation analysis, or simply peak magnitude beyond expected ranges. The results are fed into a fault classification unit to identify fault type and level.

On the other hand, it is difficult to find methodologies allowing rail-sector decision makers to choose whether to install a HMS. A ROLL2RAIL research-project deliverable provided an extensive review of the state of the art of cost assessment methods applicable in railway contexts (D4.2 [6]). The project included a number of key European rail-sector stakeholders and pointed to a lack of evidence of the economic benefits of proposed rollingstock innovations from the life cycle and rail system perspectives. The cost models, cost structures and business cases for different stakeholders were analysed (including train operating companies and integrated railways) and used to formulate specifications for a socalled Universal Cost Model (UCM). The model is described and examples of applications given in the deliverables (D4.3, D4.4, D4.5, D4.6 [6]). The UCM covers several cost categories (wheel and track maintenance, noise, energy etc.) and includes a function for unavailability costs which considers two types of unavailability: short term $Q_{1}$ and long term $Q_{2}$. The conditions associated to the vehicle which cause service unavailability are classified in two groups: operational and logistic. The former include all the different vehicle conditions related with the running gear operation which cause the unavailability; the latter include all the different maintenance conditions. The exact definition of the unavailability type is left open since it depends on the nature of the application. Examples are given in D4.3: a failure in the primary suspension causing a speed restriction and consequent delay in the service of 2 to 15 minutes is an example of $Q_{1}$; a low-speed derailment with no fatalities nor serious injuries that blocks the line causing delays larger than 15 minutes is an example of $Q_{2}$. A penalty cost is attributed to such service 
unavailability events, in a metro-related example (D4.5) respectively $€ 5 \mathrm{k}$ and $€ 1 \mathrm{M}$. The annual costs for service unavailability per trainset are the output of interest that the UCM can provide. It is modelled simply as the product of the annual probability of an unavailability event and the associated penalty cost. Procedural steps are given for the calculation of the annual unavailability costs: (1) identify conditions of vehicle which cause vehicle unavailability; (2) identify how components contribute to unavailability (this includes FTA according to standard IEC 60125 [7]); (3) calculate the unavailability costs; and (4) assessment of the trustworthiness of the calculation.

The methodology presented in this paper is intended to complement step 2 of the procedure through an FTA approach.

\section{CASE STUDY DESCRIPTION}

The case study presented in this paper is based on the Metro de Madrid type 8000 three-unit trainset (motor-trailer-motor), first series (Fig. 1). The more recent second series is about 10 years newer with many upgrades. The combined series constitute a fleet of 92 trainsets with compositions ranging from three to six pieces.

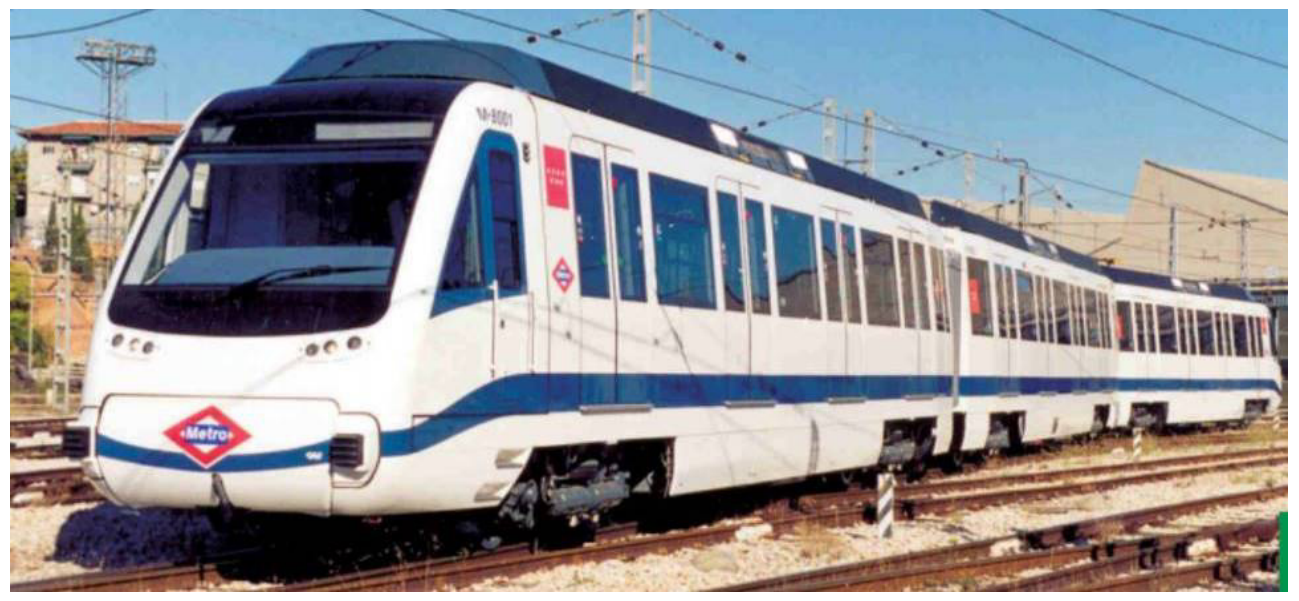

Figure 1: Metro de Madrid type 8000, first series, three-car trainset.

The second series trainset has a gearbox HMS with sensors for suspended metallic oil particles and oil/gearbox-case temperatures. Both the first and second series are equipped with pressure monitoring equipment in the secondary suspension air-springs. The first series is considered as the benchmark.

The innovation case assessed in RUN2Rail was the introduction on this trainset type of HMS for gearbox (not present in the benchmark trainset), wheelset and primary suspension.

\section{METHODOLOGY DEVELOPED AS A RESULT OF THE RESEARCH}

The methodology developed was designed to fit into ROLL2RAIL's Universal Cost Model (UCM). The annual service unavailability costs $C_{Q 1}, C_{Q 2}$, with and without CMS are calculated with the simple model of Fig. 2. The focus was on developing the FTA part for the operational unavailability. The penalty costs $Q_{1}, Q_{2}$ were taken as fixed, with values from a previous UCM application to a Metro Madrid case (D4.5 [6]). The probabilities $p_{Q 1}$, $p_{Q 2}$, as a consequence of this formulation, represent the annual probability of the occurrence 
of an event that can cause the damage quantified by $Q_{1}, Q_{2}$. The quantification of the probabilities was performed with fault trees, where the top event is the occurrence of the unavailability event. One such tree is shown in Fig. 3. Note that the fault tree starts from failure modes (FM) of items (the trainset's main sub-assemblies: axle-box ABX, gear-box GBX, wheelset WST, suspension SUS, bogie frame BGF and active suspensions systems ACT) to arrive at unavailability not of the trainset itself, but of the transport service.

$\frac{\text { probability of Top Event }}{\text { per trainset per year }}$

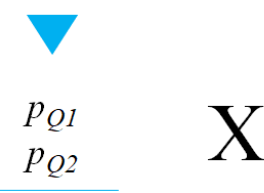

penalty cost for Top event

Q1 (5 k€) cost per trainset per year attributed to unavailability $(1,2)$

Figure 2: Unavailability cost model (ROLL2RAIL).

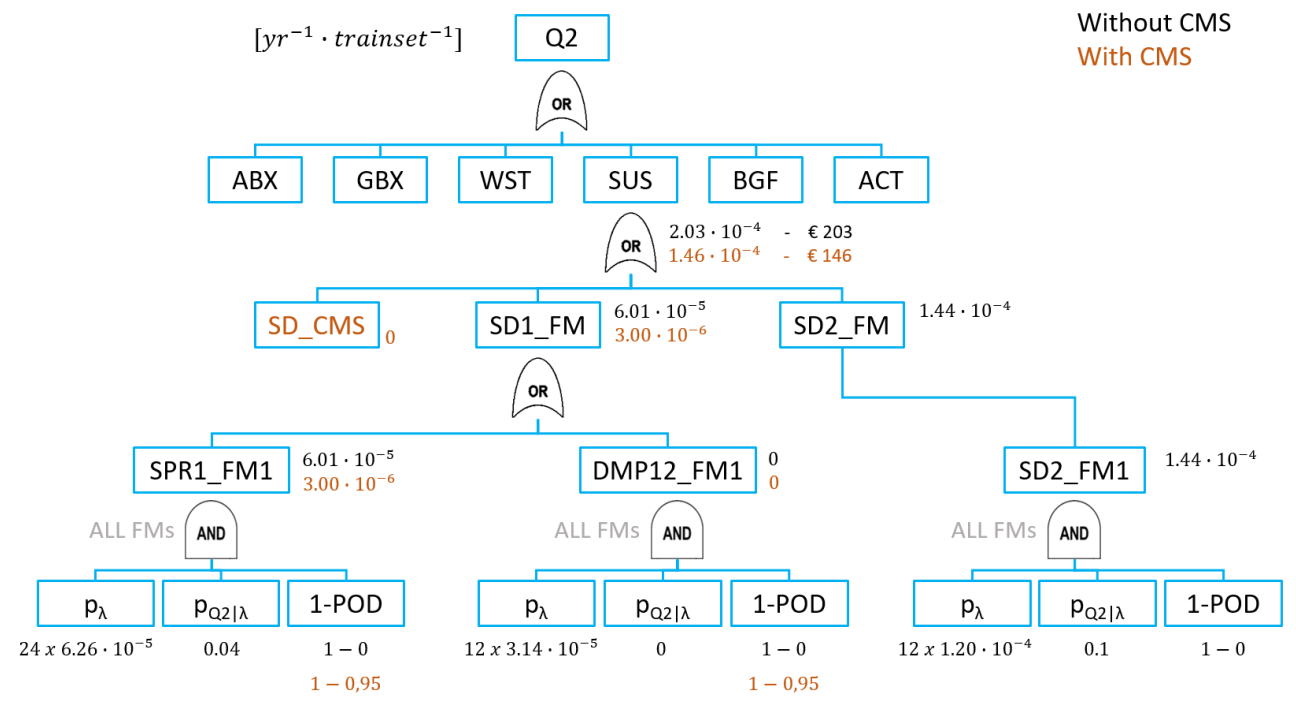

Figure 3: FTA type analysis for the quantification of the probability and cost of an unavailability event generated by the suspension system and causing $€ 1 \mathrm{M}$ in damage.

The probability of the top event is the sum of the probabilities of events in any of the sub-assemblies capable of causing the top event:

$$
\mathrm{p}_{\mathrm{Q} 2}=\mathrm{p}_{\mathrm{ABX}}+\mathrm{p}_{\mathrm{CBX}}+\mathrm{p}_{\mathrm{wST}}+\mathrm{p}_{\mathrm{sus}}+\mathrm{p}_{\mathrm{BGF}}+\mathrm{p}_{\mathrm{ACT}},
$$

in which it was assumed that $p_{B G F}=0$ since no bogie frame failures have ever been recorded in the service of type 8000 and $p_{A C T}=0$ is set because the innovative actively 
controlled suspension elements proposed in RUN2Rail are not the object of assessment here. The diagram refers to one of the items (the suspension elements). In theory, the top event is linked to suspension sub-assembly failures either because there is an actual failure in the primary or secondary springs or dampers (all failure modes) or to a failure in the "spring-damper" CMS

$$
\mathrm{p}_{\mathrm{suS}}=\mathrm{p}_{\mathrm{SD} \_\mathrm{CMS}}+\mathrm{p}_{\mathrm{SD} 1 \mathrm{~F} \mathrm{M}}+\mathrm{p}_{\mathrm{SD} 2 \mathrm{~F} \mathrm{M}} .
$$

It was assumed that such failures would not occur simultaneously, and the CMS was assigned probability zero of causing the top event. Observing for example the first element on the left (primary springs, all failure modes SPR1_FM1), the associated probability is

$$
\mathrm{p}_{\mathrm{SPR} 1 \_\mathrm{FM} 1}=\mathrm{p}_{\lambda} \cdot \mathrm{p}_{\mathrm{Q} 2 \mid \lambda} \cdot(1-\mathrm{POD}),
$$

meaning that a failure in this sub-assembly, through any one of its modes, can lead to the top event depending on:

1. its occurrence (probability of failure $\mathrm{p}_{\lambda}$ ) AND;

2. the fact that once it has occurred, it can cause an effect severe enough to generate the top event (conditional probability $\mathrm{p}_{\mathrm{Q} 2 \mid \lambda}$ ) AND;

3. that the CMS does not detect the occurrence in time for the consequences to be prevented (the complement to 1 of its probability of detection, 1-POD).

Fig. 3 also shows how the quantifications described above allow the determination of cost figures with and without CMS. The two cost figures indicated at the top (with CMS: $C_{Q 2}^{*}$, without CMS: $C_{Q 2}$ ) are obtained by multiplying the respective probability values and penalty costs (in the example $€ 1 \mathrm{M}$, type 2 unavailability) according to Fig. 2.

An indication of how much it is worth investing in the CMS is given by the present value of benefits (PVB) over the trainset's lifetime of the annual savings (difference in cost with and without CMS)

$$
P V B_{N}=\sum_{n=1}^{N} \frac{\left(C_{Q 1}^{*}+C_{Q 2}^{*}\right)-\left(C_{Q 1}+C_{Q 2}\right)}{(1+i)_{n}} .
$$

In fact this represents the present value of the future savings at the moment of investment in the CMS, considering a lifetime of $N$ years and a discount rate $i$. The overall investment for the CMS (mainly acquisition and additional maintenance costs) must be lower than this for the investment to be worthwhile.

The key aspect of the methodology is represented by the assumptions made for the three key inputs required for the probability calculations. These are discussed in the next section.

\section{ASSUMPTIONS}

\subsection{Failure rates}

Failure rates are needed for the calculation of unavailability costs according to the methodology described above. They were inspired to Metro Madrid information (number of running-gear subsystem failures, dates of entry into service for each trainset, trainset configuration) assuming the annual hours of service as in ROLL2RAIL D4.5 [6]. The values adopted in this study are shown in Table 1, note that here SUS is further subdivided into primary springs (SPR1), primary/secondary dampers (DMP12), secondary springs/ dampers (SD2). The sub-assemblies with the highest failure rates are axle-box, gearbox, 
Table 1: Assumptions for conditional probability $\mathrm{p}_{\mathrm{Q} \mid \lambda_{\mathrm{i}}}$ and return times $\mathrm{T}_{\mathrm{Ri}}$.

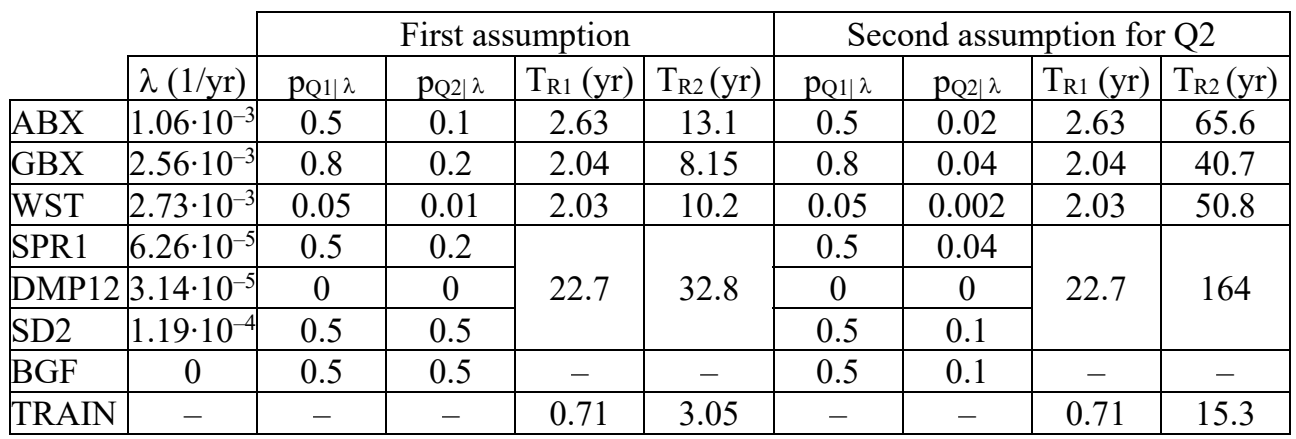

wheelset. All the other sub-assemblies have lower influence. The airsprings are considered to have the existing air-pressure CMS. The bogie frame has never had a failure since the 8000 type has been in service, so its failure rate was considered to be practically zero.

\subsection{Conditional probabilities}

The other main inputs required by the methodology are the probabilities of an unavailability event occurring given the occurrence of a failure on each specific runninggear sub-assembly. Table 1 shows a first assumption also for these values, as well as for the corresponding return times between unavailability events for a fleet of $\mathrm{N}_{\mathrm{F}}=30$ trainsets,

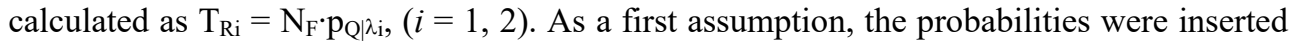
by asking questions such as, "how many axle-box failures would it take to generate a minor/major operational unavailability event?". Not knowing the exact nature of each failure, for axle-box, gearbox and suspension elements it was assumed that a large part of possible failures (60-100\%) would cause unavailability because they would be noticeable and alarming (i.e. noisy) to a degree that service could not be continued. Hot axle-boxes would mostly go unnoticed by the passengers. For the bogie frame it was assumed that an occurrence could lead with almost certainty to a disruption, however this assumption is not very influential on the results given its virtually zero failure rate. The wheelset has a relatively high failure rate, it was assumed that this is mostly due to flats and possibly the exceedance of in-service limits for geometrical parameters. Such failures would not often cause service unavailability but would be addressed at the end of scheduled service.

In order to better understand the implications of the assumptions for the conditional probabilities, a comparison was performed (Table 2) with ROLL2RAIL results taken from a Metro Madrid case with the same trainset (D4.5 [6]).

With the first assumptions of Table 1, the cost figures for unavailability $Q_{1}$ turned out to be quite similar. The $Q_{2}$ figures were, on the other hand, quite different. The ROLL2RAIL probabilities (order of $10^{-9}$ per hour, corresponding to $10^{-5}$ per year) are only a little higher than the $\sim 5 \cdot 10^{-10} 1 / \mathrm{h}$ attributed in the same ROLL2RAIL document to the probability of a catastrophic accident. The corresponding return time is of hundreds of years, which may be read that in practice during the lifetime of a metro company such an event would never occur. This leads to believe that the type of event associated to $€ 1 \mathrm{M}$ consequences for the operator is probably assumed in that report as generated by physical damage. This assumption leads to very low estimates of $C_{Q 2},<100 € /$ (year-trainset). The potential savings 
Table 2: Comparison between ROLL2RAIL and RUN2Rail results.

\begin{tabular}{|l|c|c|c|c|}
\cline { 2 - 5 } \multicolumn{1}{c|}{} & \multicolumn{2}{c|}{ ROLL2RAIL } & \multicolumn{2}{c|}{ RUN2Rail } \\
\cline { 2 - 5 } \multicolumn{1}{c|}{} & Std. wheelset & On-board lubr. & 1st assumption & 2nd assumption \\
\hline $\mathrm{p}_{\mathrm{Q} 1}(1 / \mathrm{yr})$ per trainset & $7.36 \cdot 10^{-2}$ & $1.46 \cdot 10^{-1}$ & $4.69 \cdot 10^{-2}$ & $4.69 \cdot 10^{-2}$ \\
\hline $\mathrm{p}_{\mathrm{Q} 2}(1 / \mathrm{yr})$ per trainset & $5.06 \cdot 10^{-5}$ & $7.82 \cdot 10^{-5}$ & $1.09 \cdot 10^{-2}$ & $2.18 \cdot 10^{-3}$ \\
\hline $\mathrm{C}_{\mathrm{Q} 1}(€ /(\mathrm{yr}$ trainset) $)$ & $€ 367.92$ & $€ 729.27$ & $€ 234.63$ & $€ 234.63$ \\
\hline $\mathrm{C}_{\mathrm{Q} 2}(€ /(\mathrm{yr}$ trainset $))$ & $€ 50.59$ & $€ 78.18$ & $€ 10,926.44$ & $€ 2,185.29$ \\
\hline $\begin{array}{l}\mathrm{C}_{\mathrm{Q} 1}+\mathrm{C}_{\mathrm{Q} 2}(€ /(\mathrm{yr} \\
\text { trainset)) }\end{array}$ & $€ 418.51$ & $€ 807.45$ & $€ 11,161.07$ & $€ 2,419.92$ \\
\hline $\mathrm{T}_{\mathrm{R} 1}(\mathrm{yr})$ fleet of 30 & 0.45 & 0.23 & 0.71 & 0.71 \\
\hline $\mathrm{T}_{\mathrm{R} 2}(\mathrm{yr})$ fleet of 30 & 659 & 426 & 3.05 & 15.3 \\
\hline
\end{tabular}

due to a CMS would of course be even less than these figures and would not justify a CMS. On the other hand, "assumption 1" made in RUN2Rail was assuming a strong internalisation of external costs (e.g. through penalties and lawsuits) in the event of disruptions even without physical damage, and this led to a fairly low return time (3 years).

There is a strong inverse relationship between the return time $T_{R 2}$ and the corresponding costs $C_{Q_{2}}$. If the return time is in the order of hundreds of years, then the unavailability costs (and potential savings) are very low. On the other hand return times of one to a few years will lead to costs in the order of $10,000 € /$ (year-trainset). This means that the exact definition of $Q_{2}$ is extremely important for a sound assessment of the convenience of a CMS, which also depends on service contract (penalties etc.) and behaviour of the public.

Therefore, a second assumption was made to increase the return time and obtain a total figure $C_{Q}$ of $€ 2,500$, which seemed a reasonable compromise between the different positions above, also considering the fact that Metro Madrid has actually chosen to install a gearbox CMS. In the second assumption, the probabilities were varied only for type 2 as shown in Table 1 (multiplied by 0.2 ). The return time correspondingly increased to 15 years (about two type- 2 events occurring in the fleet's lifetime). Note that with this assumption a gearbox failure would lead to a type- 2 event every 40 years, by no means a low value.

It follows from the discussion that the conditional probabilities, together with the top event definitions, are the key assumptions affecting the accuracy of the results.

\subsection{Probability of detection}

Probability of (early) detection POD should be understood as the probability of the CMS in detecting a failure that would cause unavailability sufficiently in advance to avoid it. Of course, there are no data on this since the CMS in question are still not in service. An assumption of $95 \%$ for this probability was used with the reasoning that high POD is expected from a CMS otherwise it is seen as useless.

\section{RESULTS}

Fig. 4 shows some results of the case study. Note that the savings due to the CMS are taken to descend only from unavailability cost reductions, as shown in Fig. 3 for the suspension example; the effects on other cost categories (e.g. track maintenance) are not considered. The CMS acquisition and maintenance costs are taken as a part of the necessary investment. 

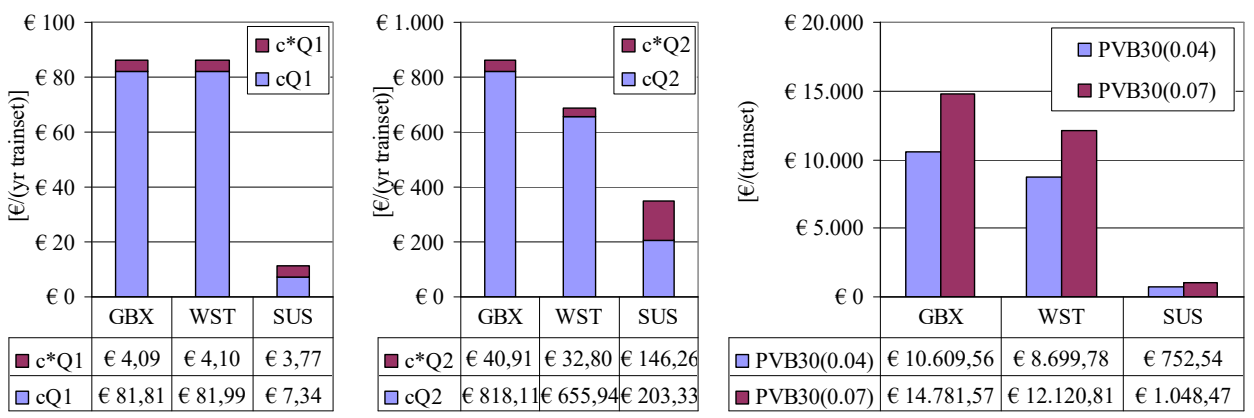

Figure 4: Annual unavailability costs per trainset with $\left(^{*}\right)$ and without CMS, and the corresponding present value of benefits.

The presence of a CMS with 95\% POD, as expected, strongly reduces the probability of occurrence of service unavailability hence the related annual costs. The present value of benefits over 30 years (trainset lifetime), calculated for discount rates of $4 \%$ and $7 \%$, corresponds to a maximum investment per trainset that would justify the adoption of a CMS. It is much lower for the suspension sub-assembly than for gearbox and wheelset. This is due to the much lower failure rates of suspension elements in the case study.

Unavailability $Q_{1}$ turns out to have a small influence, and the choices thus depend mostly on the assumptions for $Q_{2}$. This result depends on the definitions of $Q_{1}$ and $Q_{2}$ and the number of levels of the analysis (only 1 and 2).

With all the inaccuracies due to the assumptions, improvable only through the input data, particularly for the conditional probabilities, the results suggest that a gearbox CMS and a wheelset CMS would be convenient if available at less than approx. $€ 10,000$ per trainset. A suspension CMS would be convenient only at less than $€ 1,000$ per trainset. This does not seem possible at all with current market prices. This result thus supports the adoption of a gearbox CMS already implemented for the second series of the trainset.

\section{CONCLUSIONS AND RECOMMENDATIONS}

A novel methodology is presented for railway decision makers to make approximate quantitative assessments of the convenience to install a condition monitoring system for condition-based maintenance on a given sub-assembly of a metro trainset fleet, or any other railway vehicle fleet. The methodology is based on the fault tree analysis approach to quantify the probabilities of occurrence of "top events" that affect availability of the transport service. It requires definitions and monetary values for the top events (in this study two levels 1 and 2 are used) as well as assumptions or data on failure rates for the monitored sub-assembly, conditional probabilities of certain failures in generating service unavailability, and the probability of detection of which the CMS to be installed is capable.

The Metro Madrid case study results turned out to be quite sensitive to the definitions and the conditional probabilities. Therefore, the accuracy of the results is improvable in future applications following the recommendations below. The preliminary results suggest that a gearbox CMS and a wheelset CMS would be top priority in terms of convenience given their failure rates, which is line with previous Metro Madrid understanding. The return times between expected failures proved to be a useful indicator for decision making. With the case study figures, if a major unavailability event ( $€ 1 \mathrm{M}$ damage) were to be 
expected every 10 years, then the installation of a suitable CMS would be highly recommendable. The convenience still holds for return times of 40-50 years.

In the future, the methodology may be further progressed in its level of detail. Most of all, it would be important to improve the quality of the inputs by enhancing operators' databases, not initially developed to cater for such a use. The conditional probabilities could be quantified by querying operators' databases to identify how many of the recorded failures actually generated unavailability events classifiable as type 1 and type 2 , and upgrading them if the information is missing. The exact definition of the second level (long-term) unavailability $Q_{2}$ proved to be extremely important for a sound assessment of the convenience of a CMS. In fact, it is difficult to define the attributes of such an event exactly, they depend for example on the service contract and penalties associated with large disruptions, as well as on the degree to which the passengers are willing to claim reimbursement for such events. An example could be a running-gear failure that stops the train in a tunnel and requires evacuation of the train. If there are no penalties nor claims, then material damage would probably be needed to generate the $€ 1 \mathrm{M}$ amount (e.g. minor derailment). The sensitivity to this issue is high and affects the calculated benefits of a running-gear condition monitoring system.

\section{ACKNOWLEDGEMENT}

The RUN2Rail project has received funding from the Shift2Rail Joint Undertaking under the European Union's Horizon 2020 research and innovation programme under grant agreement No. 777564. The paper reflects the authors' view only and the Joint Undertaking is not responsible for any use that may be made of the information it contains.

\section{REFERENCES}

[1] EN 13306:2017, Maintenance - Maintenance terminology.

[2] Jarillo, J.M. et al., Novel technology concepts and architecture for on-board conditionbased monitoring of railway running gear: The RUN2Rail vision. Proceedings of the Institution of Mechanical Engineers, Part F: Journal of Rail and Rapid Transit, 0954409720951409, 2020.

[3] Li, C., Luo, S., Cole, C. \& Piryagin, M., An overview: modern techniques for railway vehicle on-board health monitoring systems. Vehicle System Dynamics, 55(7), pp. 1045-1070, 2017.

[4] Xu, J. \& Xu, L., Integrated System Health Management: Perspectives on Systems Engineering Techniques, Academic Press, 2017.

[5] Velletrani, F., Licciardello, R. \& Bruner, M., Intelligent wheelsets for the trains of the future: The role of in-service wheel-rail force measurement. Ingegneria Ferroviaria, 10, 2020.

[6] ROLL2RAIL public deliverables, ROLL2RAIL project, 2016. www.roll2rail.eu/Page.aspx?CAT=DELIVERABLES\&IdPage $=45291 \mathrm{e} 18-8 \mathrm{~d} 8 \mathrm{f}-4 \mathrm{fd} 6-$ 99f8-5d4b7a519b9c. Accessed on: 10 Jul. 2020.

[7] IEC 60125, Fault tree analysis (FTA). 